\title{
Lossy Mode Resonance Optical Fiber Sensor to Detect Organic Vapors
}

\author{
C. Elosua ${ }^{1}$, I. Vidondo ${ }^{1}$, F.J. Arregui', C. Bariain ${ }^{1}$, I.R. Matías ${ }^{1}$, A. Luquin ${ }^{2}$, M. Laguna ${ }^{2}$ \\ ${ }^{1}$ Public University of Navarre, Campus Arrosadia s/n E31006, Spain \\ cesar.elosua@unavarra.es \\ ${ }^{2}$ Inst de Sintesis Quimica y Catalisis Homogenea (CSIC), Plz S. Francisco s/n E50006, Spain
}

\begin{abstract}
:
A transmission sensor able to detect Volatile Organic Compounds (VOCs) has been developed using optical fiber with Plastic Cladding (PCS). Specifically, $1.5 \mathrm{~cm}$ of the cladding was removed: along this section, polymer nanolayers were deposited by means of the Layer-by-Layer method (LbL). This structure was doped with an organometallic material sensitive to VOCs. In this manner, Lossy Mode Resonances (LMRs) were induced in the transmission spectra of the sensor showing a wavelength shift as the nano structure grown. The sensing material accelerated the LMRs appearance with respect to the nanolayers without additive reported in previous works, and also, its spectral shift. Once the construction process was completed, two LMRs were observed: the first one was located at 778 $\mathrm{nm}$ and the second one at $463 \mathrm{~nm}$. In presence of ethanol vapors, the sensor spectrum varied, showing the first LMR peak a remarkable shift of $100 \mathrm{~nm}$. This behavior is reversible and was also registered for isopropanol vapors. The sensor response was analyzed as well for different concentrations of ethanol vapors.
\end{abstract}

Key words: Volatile Organic Compounds, Optical Fiber, Lossy Mode Resonances, Layer by Layer, Spectral Shift.

\section{Introduction}

Optical fiber sensors offer some interesting advantages in some specific fields, such as the ones related with the detection of VOCs. Electromagnetic immunity, remote sensing and passive nature are just some of the features that are desirable in this kind of applications. Among the different sensing schemes, sensors based on spectral shifts, not only on optical power variations, are more robust: they are selfreferenced, insensitive to optical source undesired fluctuations and to other type of artifacts related with power signal level. More specifically, LMR sensors have been reported very recently as promising devices based on wavelength shifts [1].

In this work, a sensor based on LMRs to detect VOCs is described. The resonances are induced by the LBL nanocoatings: they include an organometallic material that reduces the number of layers necessary to generate LMRs and it is also sensitive to VOCs. Spectral changes are reported for two different alcohols: ethanol and isopropanol. In the case of ethanol, the sensor was exposed to different vapor concentrations: the relative transmission spectrum was registered in each case, looking for a relationship between this parameter and the spectral shift.

\section{Experimental}

The dimensions of fiber core/cladding were $200 / 220 \mu \mathrm{m}$ respectively. The cladding was thermally removed, cleaning this segment with ethanol in order to eliminate any organic remain. Thereafter, nanolayers were deposited by means of the LbL method [2]. The polymers used were poly(allylamine hydrochloride) (PAH) and poly(acrylic acid) (PAA). $10 \mathrm{mM}$ Solutions adjusted at $\mathrm{pH} 8$ of each polymer were prepared The organometallic sensing material $\left(\left[\mathrm{Au}_{2} \mathrm{Ag}_{2}\left(\mathrm{C}_{6} \mathrm{~F}_{5}\right)_{4}\left(\mathrm{C}_{14} \mathrm{H}_{10}\right)\right]_{\mathrm{n}}\right)$ was included in the construction process by preparing a colloid with a surfactant solution (sodium dodecyl sulfate, SDS). The fiber was immersed in the solutions following this sequence: $\mathrm{PAH}-\mathrm{Colloid}-\mathrm{PAH}-$ PAA, conforming a construction cycle [3]. The fiber was rinsed in ultrapure water after each polymeric immersion to remove unabsorbed polymeric chains. Up to 7 cycles were performed to prepare the sensor.

The experimental set up is shown in Fig. 1. This configuration was used to register the evolution of the transmitted spectra along the construction process and to monitor the 
spectral shifts when the sensor was exposed to VOCs as well. The sensor is placed in a stainless steel chamber which is closed hermetically once the VOCs are injected.

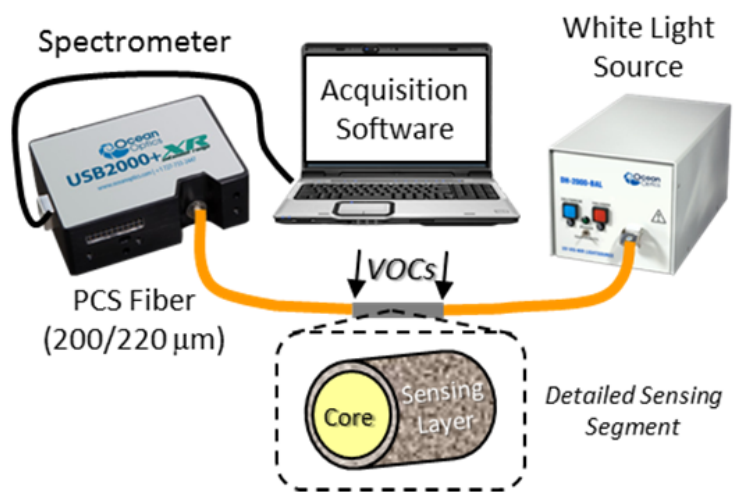

Fig 1. Experimental set up used to characterize and analyze the sensor.

\section{Results and Discussion}

All the transmission spectra registered are referenced with respect to the original spectra recorded when the cladding is removed and there is no deposition along the fiber (baseline). In order to study the construction process, the spectra were registered after every deposition cycle (plotted in Fig. 2).

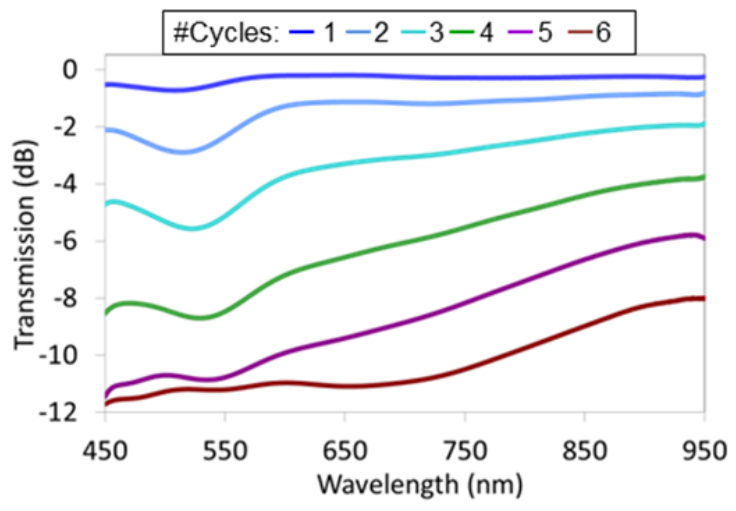

Fig . 2. Transmission spectra from the first to the sixth construction cycles.

The $1^{\text {st }}$ LMR is observable from the second cycle, showing a shift to higher wavelengths for the following cycles. A second LMR around 450 $\mathrm{nm}$ appears at the $6^{\text {th }}$ cycle. The construction process ends after the $7^{\text {th }}$ cycle: the spectrum recorded is plotted in Figure 3. In this graph,

Tthe first LMR is centered at $778 \mathrm{~nm}$, whereas the second one is at $496 \mathrm{~nm}$. In other works, up to 25 cycles were required to induce the first LMR using the same polymers of this work [3]. In this case, the refractive index of the sensing material makes the resonance conditions to be met with a minor number of cycles. The final nanoconstruction thickness is around $700 \mathrm{~nm}$.

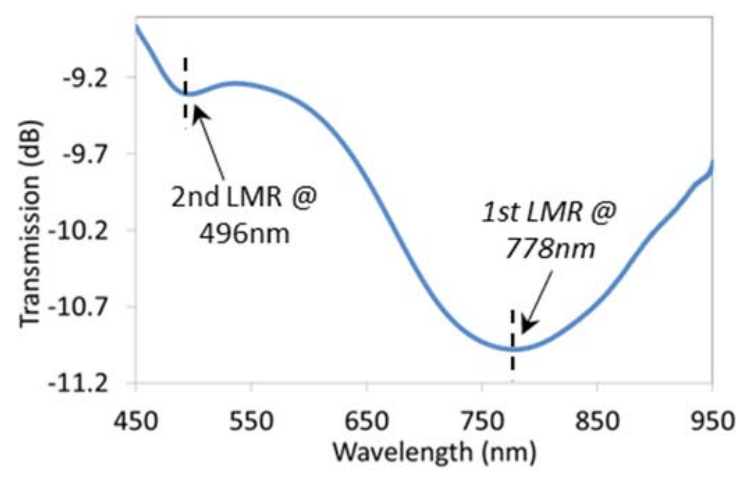

Fig. 3. Transmission spectrum once the sensor construction is finished.

The spectral location of both LMRs varies in organic vapors saturated atmospheres. The most noticeable shift is observed for the 1st LMR. In the case of ethanol, the transmission valley moves to $678 \mathrm{~nm}$ (100nm down); in the case of isopropanol, it is located at $714 \mathrm{~nm}$. This spectral shift can be observed in Fig. 4. In both cases, the spectral change is reversible. Moreover, this type of behavior might be observed also for other VOCs [4].

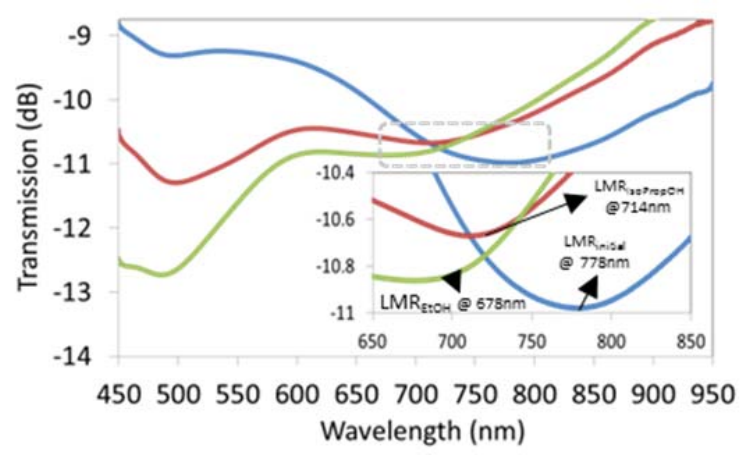

Fig. 4. Transmission spectra with no organic vapors (blue), in saturated atmospheres of isopropanol (red) and ethanol (green). In set, a detail of the wavelength shift shown by the $1^{\text {st }}$ LMR in presence of the VOCs.

The sensor was exposed to different concentrations of ethanol vapors. The final construction spectrum was considered as reference: therefore, the base line is a flat $0 \mathrm{~dB}$ line [5]. The results obtained are in Figure 5.

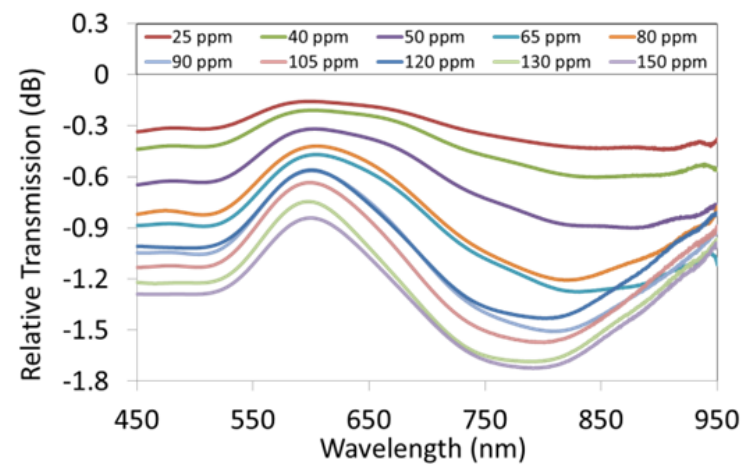

Fig. 5. Relative transmission spectra registered for different ethanol vapor concentrations ppm $(\mathrm{v} / \mathrm{V})$. 
As the concentration increases, a minimum transmission appears and moves to lower wavelengths (blue shift). Besides, it gets deeper in transmission losses. For the first concentrations, the transmission valley is very wide, but it gets narrower (and more noticeable) for higher ppm (v/v). It was found an almost linear relationship between the wavelength location of this minimum and the vapor concentration, which is shown in Figure 6.

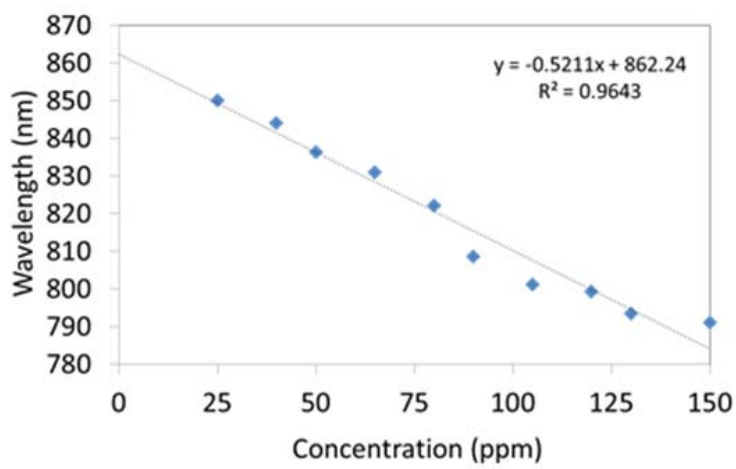

Fig. 6. Linear approximation between the relative transmission minimum wavelength and ethanol vapors concentration.

\section{Conclusions}

A sensor based on LMRs has been developed using an organometallic compound and following the LbL method. The sensing material is included along the construction process, which accelerates both the appearance and the spectral shift of the optical resonances. Actually, after 7 construction cycles 2 LMRs were present in the transmission spectrum of the sensor. The first LMR wavelength varies in saturated atmospheres with ethanol and isopropanol vapors individually. Moreover, a linear relationship is found between the spectral shift and the ethanol vapors concentration.

\section{Acknowledgments}

The authors would like to acknowledge the financial support from the Spanish Ministerio de Educación y Ciencia through projects TEC2010-17805 and TEC2010-20224-C02-01.

\section{References}

[1] I. del Villar, C. R. Zamarreño, P. Sanchez, M. Hernáez, C. F. Valdivieso, F.J. Arregui, I. R. Matias Generation of lossy mode resonances by deposition of high-refractive index coatings on uncladded multimode optical fibers, Journal of Optics 12, 095503-7, (2010)

[2] J. Goicoechea, C.R. Zamarreño, I.R. Matias, F.J. Arregui, Optical fiber $\mathrm{pH}$ sensors based on layerby-layer electrostatic self-assembled Neutral Red Sensors and Actuators B: Chemical 132, 305311, (2008).
[3] C.R. Zamarreño, M. Hernáez, I. Del Villar, I.R. Matías, F.J. Arregui, Optical fiber $\mathrm{pH}$ sensor based on lossy-mode resonances by means of thin polymeric coatings, Sensors and Actuators B: Chemical 115, 209-297, (2011).

[4] A. Luquin, C. Bariain, E. Vergara, E. Cerrada, J. Garrido, I.R. Matias, M. Laguna, Applied Organometallic Chemistry 19, 1232-1238, (2005).

[5] C. Elosua, C. Bariain, I.R. Matias, F.J. Arregui, E. Vergara, M. Laguna, Sensors and Actuators B: Chemical 13, 139-146, (2009). 